\title{
Predictors of Long-Term Psychosocial Functioning and Health-Related Quality of Life in Children and Adolescents With Prior Concussions
}

\author{
Vickie Plourde, ${ }^{1}$ Keith Owen Yeates, ${ }^{2,3,4}$ And Brian L. Brooks ${ }^{2,4,5}$ \\ ${ }^{1}$ Faculty Saint-Jean, University of Alberta, Edmonton, Alberta, Canada \\ ${ }^{2}$ Departments of Psychology, Pediatrics, and Clinical Neurosciences, University of Calgary, Calgary, Alberta, Canada \\ ${ }^{3}$ Hotchkiss Brain Institute, University of Calgary, Calgary, Alberta, Canada \\ ${ }^{4}$ Alberta Children's Hospital Research Institute, University of Calgary, Calgary, Alberta, Canada \\ ${ }^{5}$ Neurosciences Program, Alberta Children's Hospital, Calgary, Alberta, Canada
}

(Received November 3, 2017; Final Revision January 16, 2018; Accepted January 18, 2018; First Published OnLine March 21, 2018)

\begin{abstract}
Objectives: Individual differences in long-term psychosocial functioning after concussions in children and adolescents are poorly understood. The aim of the study was to investigate potential predictors of long-term psychosocial functioning and health-related quality of life in youth after prior concussion. Methods: Participants $(N=75$; mean age $=14.3$ years old; $52 \%$ girls) with one prior concussion $(n=24)$, multiple prior concussions $(n=24)$, or a prior orthopedic injury and no concussion $(n=27)$ were seen on average 2.7 years after their most recent injury. Psychosocial functioning was assessed using the self-report versions of the Behavior Assessment System for Children (BASC-2; Anxiety and Depression scales only), the Strengths and Difficulties Questionnaire, and the Pediatric Quality of Life Inventory TM 4.0. Pre-existing conditions (attention problems, learning difficulties, mood concerns, anxiety concerns, and migraines) were reported by parents using a checklist and examined as predictors of long-term functioning. Other potential predictors included age at testing, sex, time between most recent injury and testing, and number of prior concussions. Results: The groups did not differ significantly on long-term psychosocial functioning. Moreover, only pre-existing mood concerns or attention problems significantly predicted psychosocial adjustment. Conclusions: Children's functioning before a concussion is critical to understanding outcome. Pre-injury attention and mood concerns should be assessed in clinical settings to prevent and treat long-term psychosocial problems after concussion. (JINS, 2018, 24, 540-548)
\end{abstract}

Keywords: Concussion, Pre-existing conditions, Childhood, Adolescence, Pediatric, Long-term functioning

\section{INTRODUCTION}

Post-concussive symptoms, including physical, cognitive, emotional, and fatigue or sleep-related symptoms (Merritt \& Arnett, 2014), commonly occur for a short-term period after sustaining a concussion (Lovell et al., 2006; Sady, Vaughan, \& Gioia, 2014). Although the majority of children and adolescents will return to baseline functioning within a month post-injury (Davis et al., 2017; Zemek et al., 2016), recovery duration may vary between individuals and be longer for a small minority of children (Barlow, 2016; Barlow et al., 2010; Iverson, 2010; Iverson \& Schatz, 2015).

Research has advanced our understanding of individual differences in children and adolescents recovering from

Vickie Plourde, Faculty Saint-Jean, University of Alberta, 8406 Rue Marie-Anne Gaboury Northwest, Edmonton, AB, Canada, T6C 4G9. E-mail: plourde@ualberta.ca a concussion, by identifying predictors of post-concussive symptoms (Davis et al., 2017; Iverson et al., 2017; Zemek, Farion, Sampson, \& McGahern, 2013). For instance, sex (i.e., being female), age (i.e., being an adolescent), health (i.e., headaches, pain), psychological (i.e., anxiety, depression, somatization), and neurodevelopmental (i.e., attention or behavior problems, learning difficulties) conditions existing before the injury have been shown to predict post-concussive symptoms from 1 to 3 months post-injury (Bernard, Ponsford, McKinlay, McKenzie, \& Krieser, 2016; Grubenhoff et al., 2016; Morgan et al., 2015; Ponsford et al., 1999; Root et al., 2016; Yeates et al., 1999; Zemek et al., 2016) to up to 12 months post-injury (McNally et al., 2013; Yeates et al., 2012). The number of previous concussions (Morgan et al., 2015; Zuckerman et al., 2016) or having a prior concussion with symptoms lasting for more than a week (Zemek et al., 2016) have also been shown to predict a prolonged recovery 1 to 3 months post-concussion. 
Therefore, the evidence to date highlights the importance of a multidimensional biopsychosocial framework and of considering both injury-related and non-injury related factors when predicting the outcomes of children with concussion. However, the predictors of concussion recovery in children and adolescents have not yet been investigated more than a year after concussion, and the relationship between multiple concussions and long-term negative health outcomes is uncertain (Institute of Medicine - IOM \& National Research Council - NRC, 2014; Catroppa et al., 2017; Sariaslan, Sharp, Onofrio, Larsson, \& Fazel, 2016; Solomon, Kuhn, \& Zuckerman, 2016). Moreover, studies of predictors of recovery from concussion in childhood and adolescence have focused mainly on post-concussive symptoms and have not considered broader domains of psychosocial functioning, such as overall emotional or behavioral adjustment or healthrelated quality of life. Indeed, difficulties in these different domains can arise acutely following a concussion, in association with acute or persistent post-concussive symptoms (Emery et al., 2016; Mccauley et al., 2012; Novak et al., 2016; Russell et al., 2017; Stazyk, DeMatteo, Moll, \& Missiuna, 2017).

The goal of the current study was to document and identify predictors of long-term psychosocial functioning in children and adolescents several years after a prior concussion. The goal was to determine if certain injury and non-injury factors in children and adolescents, such as number of previous concussions or pre-existing conditions, play an important role in predicting psychosocial functioning in the longer term post-injury. The assumption was that the findings would provide new knowledge relevant to working clinically with children and adolescents who report long-term psychosocial problems following concussions. More specifically, the study had two objectives: (1) to compare long-term psychosocial functioning and healthrelated quality of life of children and adolescents who had one concussion, multiple concussions, or an orthopedic injury but no concussion; and (2) to determine which of the following factors-age, sex, number of previous concussions, and pre-existing migraines, attention problems, learning difficulties, depression, and anxiety symptomswere predictors of their psychosocial functioning long after their last injury.

\section{METHOD}

\section{Participants}

The study included three groups of participants (total $N=75)$ : children with one concussion only $(n=24)$, children with multiple concussions $(n=24)$, or children with an orthopedic injury (OI) and no prior concussion $(n=27)$. Recruitment involved inviting children who participated in previous concussion research projects in the emergency department or in previous research in a sports injury prevention program for children with orthopaedic injuries. Several eligible individuals declined participation $(N=112$;
Mean age $=15.36 ; S D=2.09 ; 36$ girls, 76 boys; 42 children with OI; 59 children with one concussion only, and 11 children with multiple concussions). Individuals who declined were significantly older, $F(1,186)=8.655$, $p=.004, \eta_{\mathrm{p}}^{2}=.045$, included a greater number of male participants, $\chi^{2}(1)=7.374, \quad p=.007, \quad r_{\varphi}=.199$, and had proportionally fewer children with multiple concussions, $\chi^{2}(2)=16.160, p<.001, r_{\varphi}=.294$, compared to participants in the study.

Participants were between 8 and 19 years of age. They and their parents had to understand and speak English to be able to complete questionnaires. All groups were screened for the following exclusion criteria: past hospitalization for a psychiatric condition; history of substance abuse or neurological disorder (e.g., seizures, hydrocephalus); or visual, hearing, motor, or language skills that prevented testing. Children and adolescents with learning disabilities, attention problems, anxiety, or depression were not excluded.

Participants in the groups with single or multiple concussions sustained their only (single concussion group) or most recent concussion (multiple concussions group; mean number of concussions $=3.04 ; S D=1.65$; range $=2-8$ ) at least 6 months before enrolment. Concussion was defined by a Glasgow Coma Scale score between 13 and 15, duration of loss of consciousness less than $30 \mathrm{~min}$, and/or duration of post-traumatic amnesia of less than $24 \mathrm{hr}$ at the time of injury), and had to be diagnosed by a healthcare professional. The OI group consisted of children who never had a concussion but presented to a healthcare professional for management of an orthopedic injury to the thorax, upper extremity, or lower extremity, consistent with an Abbreviated Injury Scale score of 3 or less (McNally et al., 2013).

\section{Measures}

\section{Pre-existing conditions checklist}

Parents were asked to report the number of previous concussions or OIs sustained by their child, and to complete the following questions (yes/no) based on their recall of their child before any injuries: non-migraine headaches; concerns about attention or diagnosed attention disorder; concerns about learning or diagnosed learning disorder; concerns about low mood, sadness, or depression; concerns about worrying or anxiety.

\section{Behavior Assessment System for Children (BASC $C_{-2}^{T M}$}

Children rated their emotional and behavioral adjustment using the age-appropriate, self-report versions of the Anxiety and Depression subscales of the Behavior Assessment System for Children (BASC-2; Reynolds \& Kamphaus, 2004) questionnaire. The BASC-2 has shown satisfactory reliability and validity (Reynolds \& Kamphaus, 2004). Scores for both subscales were standardized (T scores) with general population age/sex norms. 


\section{Strengths and Difficulties Questionnaire (SDQ)}

The SDQ is a 25-item rating scale that assesses psychosocial adjustment (Goodman, 1997). Five items are included in each of the following scales (score range from 0 to 10): (1) emotional symptoms, (2) conduct problems, (3) hyperactivity/ inattention, (4) peer relationship problems, and (5) prosocial behavior. A total difficulties score composed of 20 items (scales 1 to 4) is also derived (score range from 0 to 40). A self-report version is available for use with children aged from 11 to 17 (Goodman, Meltzer, \& Bailey, 1998); however, studies have shown it is valid for use with younger children or older adolescents (Bøe, Hysing, Skogen, \& Breivik, 2016; Curvis, Mcnulty, \& Qualter, 2014; Muris, Meesters, Eijkelenboom, \& Vincken, 2004; Roy, Veenstra, $\&$ Clench-Aas, 2008). The self-report version was used for all participants in this study. The SDQ has shown satisfactory reliability and validity (Bourdon, Goodman, \& Rae, 2005; Goodman et al., 1998; Goodman, 2001).

\section{Pediatric Quality of Life Inventory TM Version 4.0 (PedsQL TM)}

The PedsQL is a 23-item questionnaire that measures healthrelated quality of life (Varni, Seid, \& Kurtin, 2001; Varni, Burwinkle, Seid, \& Skarr, 2003; Varni, Seid, \& Rode, 1999). Eight items are included in the physical functioning scale, and five items are included in each of the emotional, social, and school functioning scales. A total score is derived, as well as a physical health summary score ( 8 items: physical scale) and a psychosocial health summary score (15 items: emotional, social, and school scales) (all scored to a common metric, $0=100$ ), with higher scores representing better health-related quality of life. Age-appropriate self-report versions were administered to the participants. Items are the same between versions but the wording slightly differs. Reliability and validity of the PedsQL is well established (Varni et al., 2001, 2003, 1999).

\section{Procedure}

The study was observational and cross-sectional, and data collection was conducted in a tertiary care children's hospital. On the day of testing, children provided assent and parents provided consent to participate to the study after having it explained to them. Then, children completed the assessment with a research assistant. The research was approved by the University of Calgary Conjoint Health Research Ethics Board.

\section{Analyses}

Statistical analyses were completed using IBM's Statistical Package for the Social Sciences, version 24 (IBM Corporation, 2013). Because of the positive skewness of some variables' distributions, square root transformations were applied to three PedsQL scores (physical functioning, social functioning, and physical health summary scores), logarithmic transformations were applied to the number of concussions and two SDQ scores (conduct problems and peer relationship problems scales), and a reciprocal transformation was applied to the depression scale from the BASC-2.

Chi-square $\left(\chi^{2}\right)$ likelihood ratio analyses were conducted to compare the three groups (participants with OI, single concussion, or multiple concussions) on categorical demographic variables as well as linear-by-linear association chi-square tests to compare groups on pre-existing conditions. Analyses of variance (ANOVAs) were conducted to compare groups on age at testing and time between the injury and testing, as well as on BASC-2, SDQ, and PedsQL scores. Bonferroni's post hoc tests were performed if ANOVAs were significant. Effect sizes were reported using Phi $\left(r_{\varphi}\right)$ for chi-square tests, interpreted as small $\left(r_{\varphi}=.1\right)$, medium $\left(r_{\varphi}=.3\right)$, or large $\left(r_{\varphi}=.5\right)$; partial eta squared $\left(\eta_{\mathrm{p}}^{2}\right)$ for ANOVAs, interpreted as small $\left(\mathrm{\eta}_{\mathrm{p}}^{2}=.01\right)$, medium $\left(\mathrm{\eta}_{\mathrm{p}}^{2}=.06\right)$, or large $\left(\mathrm{\eta}_{\mathrm{p}}{ }^{2}=.14\right)$; and Cohen's $d$ for post hoc tests, interpreted as small $(d=.20)$, medium $(d=.50)$, or large $(d=.80)$ (Cohen, 1992). Finally, standard multiple regression analyses using pairwise deletion were conducted to examine predictors of long-term functioning in children. All predictors were entered simultaneously in these models. Predictors included in the regression analyses were selected based on previous theoretical and empirical support, as well as available data. To control for multiple tests, a conservative $p$ level (.01) was set $a$ priori for all analyses.

\section{RESULTS}

Demographics are reported in Table 1, divided by group (participants with OI, single concussion, or multiple concussions). The groups did not differ significantly on sex, $\chi^{2}(2)=.3 .305, p=.192, r_{\varphi}=.208$, or race, $\chi^{2}(6)=8.859$, $p=.182, r_{\varphi}=.332$. They also did not differ significantly on time between injury and testing, $F(2,58)=.621, p=.541$, $\eta_{\mathrm{p}}^{2}=.021$, or age at testing, $F(2,72)=4.125, p=.020$, $\eta_{\mathrm{p}}{ }^{2}=.103$. Finally, most parents reported holding a college (mother: $29.3 \%$; father: $40.0 \%$ ) or a university degree (mother: 61.3\%; father: $46.7 \%$ ), with no significant differences between groups for mother's, $\chi^{2}(4)=3.699, p=.448, r_{\varphi}=.223$, or father's education, $\chi^{2}(6)=7.860, p=.249, r_{\varphi}=.305$.

The groups did not differ significantly in the proportions reporting pre-existing attention problems, $\chi^{2}(1)=3.569$, $p=.059, r_{\varphi}=.244$, pre-existing learning difficulties, $\chi^{2}(1)=5.753, p=.016, r_{\varphi}=.284$, pre-existing mood concerns, $\chi^{2}(1)=.902, p=.342, r_{\varphi}=.240$, pre-existing anxiety concerns, $\chi^{2}(1)=.471, p=.492, r_{\varphi}=.097$, or pre-existing migraines, $\chi^{2}(1)=1.300, p=.254, r_{\varphi}=.207$.

The groups also did not differ significantly on BASC-2 anxiety and depression scores, SDQ scores, or PedsQL scores at a mean of 2.7 years post-injury (Table 2). We performed additional analyses controlling for demographics and preexisting conditions for which the groups showed medium effect size differences (i.e., race, age at testing, father's education, and pre-existing learning problems). Results were essentially unchanged after controlling for these confounding variables. 
Table 1. Sample characteristics

\begin{tabular}{lccc}
\hline \hline Groups & Orthopedic injury & Single concussion & Multiple concussions \\
\hline Sex (\% Boys) & 51.90 & 33.30 & 58.30 \\
$n$ & 27 & 24 & 24 \\
Race (\% Caucasian) & 85.20 & 79.20 & 95.80 \\
$n$ & 27 & 24 & 24 \\
Age [M(SD)] & $14.00(2.93)$ & $13.33(2.72)$ & $15.55(2.59)$ \\
(range) & $(8.46-19.11)$ & $(8.54-18.48)$ & $(8.77-18.65)$ \\
$n$ & 27 & 24 & 24 \\
Time injury-testing (in months) & $28.90(29.68)$ & $35.92(14.23)$ & $32.03(14.06)$ \\
(range) & $(4.27-130.66)$ & $(11.99-55.46)$ & $(10.87-73.46)$ \\
$n$ & 18 & 22 & 21 \\
Pre-existing attention (\% Yes) & 14.80 & 16.70 & 37.50 \\
$n$ & 27 & 24 & 24 \\
Pre-existing learning (\% Yes) & 3.70 & 20.80 & 29.20 \\
$n$ & 27 & 24 & 24 \\
Pre-existing mood concerns $(\%$ Yes) & 25.93 & 4.35 & 16.67 \\
$n$ & 27 & 23 & 24 \\
Pre-existing anxiety concerns (\% Yes) & 29.63 & 30.44 & 20.83 \\
$n$ & 27 & 23 & 24 \\
Pre-existing migraines (\% Yes) & 11.11 & 0 & 4.17 \\
$n$ & 27 & 24 & 24 \\
\hline \hline
\end{tabular}

Notes. $M=$ mean; $S D=$ standard deviation.

Multiple regression analyses were completed using the full sample (all three groups) and pairwise deletion to examine predictors of long-term functioning post-injury (Table 3).
The number of concussions was not a significant predictor of long-term anxiety or depression (BASC-2), overall psychological/behavioral functioning (SDQ), or overall

Table 2. ANOVAs comparing the three groups on long-term functioning post-injury

\begin{tabular}{|c|c|c|c|c|c|c|c|}
\hline Variables & $\begin{array}{c}\text { OI } \\
M(S D)\end{array}$ & $\begin{array}{c}\text { Single C } \\
M(S D)\end{array}$ & $\begin{array}{c}\text { Multiple C } \\
\qquad M(S D)\end{array}$ & $F$ & $d f$ & $p$-Value & $\mathrm{\eta}_{\mathrm{p}}^{2}$ \\
\hline BASC- $2^{\text {a }}$ Anxiety & $49.69(11.16)$ & $47.17(10.16)$ & $49.92(9.20)$ & .540 & 2,73 & .585 & .015 \\
\hline$n$ & 26 & 24 & 24 & & & & \\
\hline BASC- $2^{\mathrm{a}}$ Depression $^{\mathrm{b}}$ & $48.12(10.87)$ & $46.71(9.12)$ & $45.63(4.80)$ & .221 & 2,73 & .802 & .006 \\
\hline$n$ & 26 & 24 & 24 & & & & \\
\hline $\mathrm{SDQ}^{\mathrm{c}}$ total difficulties & $9.59(4.84)$ & $9.63(6.45)$ & $10.38(4.25)$ & .174 & 2,74 & .840 & .005 \\
\hline$n$ & 27 & 24 & 24 & & & & \\
\hline Emotional & $2.37(2.48)$ & $2.75(2.33)$ & $2.88(1.83)$ & .355 & 2,74 & .702 & .010 \\
\hline Conduct problems ${ }^{\mathrm{d}}$ & $1.56(1.40)$ & $1.67(1.99)$ & $1.25(1.03)$ & .230 & 2,74 & .795 & .006 \\
\hline Hyperact/inattention & $3.78(2.21)$ & $3.38(2.95)$ & $4.58(2.52)$ & 1.389 & 2,74 & .256 & .037 \\
\hline Peer relationship $^{\mathrm{d}}$ & $1.89(1.91)$ & $1.83(1.99)$ & $1.67(1.31)$ & .020 & 2,74 & .981 & .001 \\
\hline Prosocial behavior & $8.04(1.53)$ & $8.29(1.85)$ & $8.21(1.69)$ & .152 & 2,74 & .860 & .004 \\
\hline PedsQL ${ }^{\mathrm{c}}$ total & $78.10(13.98)$ & $81.12(13.92)$ & $76.90(14.36)$ & .844 & 2,73 & .434 & .023 \\
\hline$n$ & 26 & 24 & 24 & & & & \\
\hline Physical health SS & $84.26(13.06)$ & 87.63 (9.99) & $81.64(16.77)$ & .450 & 2,73 & .640 & .013 \\
\hline Physical $^{\mathrm{e}}$ & $5.04(4.18)$ & $3.96(3.20)$ & $5.88(5.37)$ & 1.179 & 2,73 & .313 & .032 \\
\hline Psychosocial health SS & $74.81(15.59)$ & $77.47(17.86)$ & $74.38(14.41)$ & .496 & 2,73 & .611 & .014 \\
\hline School & $5.12(3.45)$ & $4.67(3.71)$ & $6.88(5.16)$ & .772 & 2,73 & .466 & .021 \\
\hline Emotional & $6.38(4.08)$ & $5.33(4.07)$ & $5.75(3.01)$ & .545 & 2,73 & .582 & .015 \\
\hline Social $^{\mathrm{e}}$ & $3.46(2.90)$ & $3.50(4.72)$ & $2.42(2.30)$ & 1.179 & 2,73 & .313 & .032 \\
\hline
\end{tabular}

Notes. $\mathrm{OI}=$ orthopedic injury; $\mathrm{C}=$ concussion; $\mathrm{BASC}-2=$ Behavior Assessment System for Children; $\mathrm{SDQ}=$ Strengths and Difficulties questionnaire; PedsQL = Pediatric Quality of Life Inventory; $\mathrm{SS}=$ Summary score; $\eta_{\mathrm{p}}^{2}=$ Partial eta squared; $F=F$ distribution; $d f=$ degrees of freedom; $p=$ probability; $n=$ number of cases; $M=$ mean; $S D=$ standard deviation.

${ }^{\mathrm{a}} \mathrm{T}$-scores reported.

${ }^{\mathrm{b}}$ Reciprocal transformation used for the ANOVA.

${ }^{\mathrm{c}}$ Raw scores were reported for all SDQ and PedsQL scores.

${ }^{\mathrm{d}}$ Logarithmic transformed scores used for the ANOVAs; $* p<.01$

${ }^{\mathrm{e}} \mathrm{Square}$ root transformed scores used for the ANOVAs; $* p<.01$. 
Table 3. Predictors of long-term psychosocial functioning and health-related quality of life

\begin{tabular}{|c|c|c|c|c|c|c|c|c|}
\hline \multirow[b]{2}{*}{$\begin{array}{l}\text { Predictors } \\
n\end{array}$} & \multicolumn{2}{|c|}{ BASC-2 Anxiety } & \multicolumn{2}{|c|}{ BASC-2 Depression $^{\text {a }}$} & \multicolumn{2}{|c|}{ SDQ total score } & \multicolumn{2}{|c|}{ PedsQL total score } \\
\hline & $\begin{array}{l}B(S E) \\
n=74\end{array}$ & $\beta$ & $\begin{array}{l}B(S E) \\
n=74\end{array}$ & $\beta$ & $\begin{array}{l}B(S E) \\
n=75\end{array}$ & $\beta$ & $\begin{array}{l}B(S E) \\
n=74\end{array}$ & $\beta$ \\
\hline $\begin{array}{l}\text { Sex } \\
n=75\end{array}$ & $-2.82(2.79)$ & -.14 & $<.001(.001)$ & -.02 & $.24(1.34)$ & .02 & $-.98(3.80)$ & -.04 \\
\hline $\begin{array}{l}\text { Age at testing } \\
n=75\end{array}$ & $.22(.45)$ & .06 & $<.001(<.001)$ & -.14 & $.04(.22)$ & .02 & $-.22(.61)$ & -.05 \\
\hline $\begin{array}{l}\text { Time since last injury } \\
n=61\end{array}$ & $.10(.07)$ & .20 & $<.001(<.001)$ & .13 & $-.003(.04)$ & -.01 & $-.08(.10)$ & -.12 \\
\hline $\begin{array}{l}\# \text { of concussions }{ }^{\mathrm{b}} \\
n=75\end{array}$ & $3.77(5.45)$ & .09 & $<.001(.001)$ & .02 & $3.47(2.62)$ & .17 & $-8.69(7.44)$ & -.16 \\
\hline $\begin{array}{l}\text { Pre-attention } \\
n=75\end{array}$ & $3.76(3.48)$ & .16 & $.002(.001)$ & .25 & $4.67(1.68)$ & $.38 *$ & $-7.93(4.75)$ & -.24 \\
\hline $\begin{array}{l}\text { Pre-learning } \\
n=75\end{array}$ & $-4.79(3.80)$ & -.18 & $-.001(.001)$ & -.09 & $-3.21(1.83)$ & -.24 & $7.91(5.18)$ & .22 \\
\hline $\begin{array}{l}\text { Pre-mood } \\
n=74\end{array}$ & $6.99(4.40)$ & .26 & $.004(.001)$ & $.53 *$ & $3.84(2.12)$ & .27 & $-13.71(6.01)$ & -.36 \\
\hline $\begin{array}{l}\text { Pre-anxiety } \\
n=74\end{array}$ & $4.10(3.48)$ & .18 & $<.001(.001)$ & .02 & $2.70(1.68)$ & .23 & $-1.41(4.75)$ & -.05 \\
\hline $\begin{array}{l}\text { Pre-migraines } \\
n=75\end{array}$ & $2.87(6.16)$ & .06 & $.001(.002)$ & .06 & $1.57(2.97)$ & .07 & $-2.55(8.40)$ & -.04 \\
\hline$R^{2}$ & .264 & & .462 & & .346 & & .278 & \\
\hline$F$ & 1.996 & & $4.771 *$ & & $2.940 *$ & & 2.137 & \\
\hline
\end{tabular}

Notes. BASC-2 = Behavior Assessment System for Children; SDQ = Strengths and Difficulties questionnaire; PedsQL $=$ Pediatric Quality of Life Inventory; $B=$ Unstandardized regression coefficients; $S E=$ standard error; $\beta=$ Standardized regression coefficients; $R^{2}=$ multiple correlation squared; $F=F$ distribution; $n=$ number of cases.

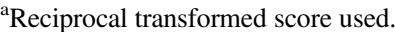

${ }^{\mathrm{b}}$ Logarithmic transformed scores used; ${ }^{*} p<.01$.

health-related quality of life (PedsQL). Instead, pre-existing attention problems significantly predicted higher overall psychological/behavioral problems, whereas pre-existing mood concerns significantly predicted higher depression symptoms.

To further investigate predictors of psychosocial functioning and health-related quality of life, additional multiple regression analyses were performed on the five subscales of the SDQ (Table 4) and the five subscales of the PedsQL (Table 5). For the SDQ, only pre-existing attention problems was a significant predictor of higher conduct problems and hyperactivity/inattention long-term after injury. No significant pre-injury predictors were identified for emotional symptoms, peer relationship problems, or prosocial behavior long after injury. On the PedsQL, no significant predictors were identified for any of the five subscales (PedsQL physical health summary score and psychosocial health summary score; school, emotional, and social subscales).

\section{DISCUSSION}

The long-term impact of multiple concussions in youth is a significant concern, but has been the topic of few studies to date. The present study compared long-term psychosocial functioning and health-related quality of life of children and adolescents who sustained one concussion, multiple concussions, or an orthopedic injury (and no concussion), and sought to identify predictors of long-term functioning in this population.

We found no significant differences between groups on current psychosocial functioning or health-related quality of life. These results are encouraging, as they suggest that children and adolescents with multiple concussions do not report worse global psychosocial functioning than children with single concussions or with injuries not involving the head. Previous studies of concussion recovery have provided mixed results (Emery et al., 2016), showing higher levels of behavior or psychological problems post-injury in some cases (Basson et al., 1991; Hawley, Ward, Magnay, \& Long, 2004; Luis \& Mittenberg, 2002) but not in others (BarkerCollo, 2007; Max et al., 2004; O'Connor et al., 2012). Previous studies also have shown poorer health-related quality of life in children with concussion compared with healthy controls up to 12 months post-injury (Moran et al., 2012; Novak et al., 2016; Yeates, Kaizar, et al., 2012). These divergent results may reflect the heterogeneity between studies in how concussion was defined, the period of time between the last concussion and assessment of outcomes, as well as the interval between multiple concussions (IOM \& NRC, 2014).

Additionally, the number of concussions was not a significant predictor of long-term psychosocial functioning 
Table 4. Predictors of long-term psychosocial symptoms (SDQ) divided by scales

\begin{tabular}{|c|c|c|c|c|c|c|c|c|c|c|}
\hline \multirow[b]{2}{*}{$\begin{array}{l}\text { Predictors } \\
n\end{array}$} & \multicolumn{2}{|c|}{ Emotional } & \multicolumn{2}{|c|}{ Conduct problems ${ }^{\mathrm{a}}$} & \multicolumn{2}{|c|}{ Hyperact/inattention } & \multicolumn{2}{|c|}{ Peer relationship ${ }^{a}$} & \multicolumn{2}{|c|}{ Prosocial behavior } \\
\hline & $\begin{array}{l}B(S E) \\
n=75\end{array}$ & $\beta$ & $\begin{array}{l}B(S E) \\
n=75\end{array}$ & $\beta$ & $\begin{array}{l}B(S E) \\
n=75\end{array}$ & $\beta$ & $\begin{array}{l}B(S E) \\
n=75\end{array}$ & $\beta$ & $\begin{array}{l}B(S E) \\
n=75\end{array}$ & $\beta$ \\
\hline $\begin{array}{l}\text { Sex } \\
n=75\end{array}$ & $.87(.57)$ & .20 & $-.02(.06)$ & -.03 & $-.27(.70)$ & -.05 & $-.02(.08)$ & -.04 & $.76(.47)$ & .23 \\
\hline $\begin{array}{l}\text { Age at testing } \\
n=75\end{array}$ & $-.003(.09)$ & -.004 & $-.01(.01)$ & -.12 & $.15(.11)$ & .17 & $-.001(.01)$ & -.01 & $.11(.08)$ & .18 \\
\hline $\begin{array}{l}\text { Time since last injury } \\
n=61\end{array}$ & $.02(.02)$ & .21 & $.001(.002)$ & .06 & $-.03(.02)$ & -.21 & $<.001(.002)$ & .01 & $-.01(.01)$ & -.08 \\
\hline $\begin{array}{l}\# \text { of concussions }{ }^{\mathrm{a}} \\
n=75\end{array}$ & $1.54(1.11)$ & .18 & $.03(.12)$ & .03 & $1.29(1.37)$ & .13 & $.14(.15)$ & .14 & $-.37(.91)$ & -.06 \\
\hline $\begin{array}{l}\text { Pre-attention } \\
n=75\end{array}$ & $.31(.71)$ & .06 & $.28(.08)$ & $.50 *$ & $2.63(.87)$ & $.43^{*}$ & $-.08(.10)$ & -.13 & $-1.48(.58)$ & -.37 \\
\hline $\begin{array}{l}\text { Pre-learning } \\
n=75\end{array}$ & $-.01(.77)$ & -.002 & $-.22(.08)$ & -.36 & $-1.25(.95)$ & -.19 & $-.09(.11)$ & -.13 & $.96(.64)$ & .22 \\
\hline $\begin{array}{l}\text { Pre-mood } \\
n=74\end{array}$ & $1.17(.89)$ & .20 & $.09(.09)$ & .14 & $.59(1.10)$ & .09 & $.24(.12)$ & .33 & $-.02(.74)$ & -.004 \\
\hline $\begin{array}{l}\text { Pre-anxiety } \\
n=74\end{array}$ & $1.12(.71)$ & .23 & $.12(.08)$ & .23 & $.26(.87)$ & .04 & $.11(.10)$ & .18 & $-.23(.58)$ & -.06 \\
\hline $\begin{array}{l}\text { Pre-migraines } \\
n=75\end{array}$ & $.75(1.25)$ & -.08 & $-.16(.13)$ & -.16 & $1.69(1.55)$ & .15 & $-.12(.17)$ & -.10 & $-1.10(1.03)$ & -.15 \\
\hline$R^{2}$ & .366 & & .362 & & .277 & & .185 & & .238 & \\
\hline$F$ & $3.207 *$ & & $3.152 *$ & & 2.128 & & 1.258 & & 1.738 & \\
\hline
\end{tabular}

Notes. SDQ = Strengths and Difficulties questionnaire; $B=$ unstandardized regression coefficients; $S E=$ standard error; $\beta=$ standardized regression coefficients; $R^{2}=$ multiple correlation squared; $F=F$ distribution; $n=$ number of cases;

${ }^{a}$ Logarithmic transformed scores used; $* p<.01$.

Table 5. Predictors of long-term health-related quality of life (PedsQL) divided by scales

\begin{tabular}{|c|c|c|c|c|c|c|c|c|c|c|}
\hline \multirow[b]{2}{*}{$\begin{array}{l}\text { Predictors } \\
n\end{array}$} & \multicolumn{2}{|c|}{ Physical health $^{\mathrm{a}}$} & \multicolumn{2}{|c|}{ Psychosocial health } & \multicolumn{2}{|l|}{ School } & \multicolumn{2}{|c|}{ Emotional } & \multicolumn{2}{|l|}{ Social $^{\mathrm{a}}$} \\
\hline & $\begin{array}{l}B(S E) \\
n=75\end{array}$ & $\beta$ & $\begin{array}{l}B(S E) \\
n=75\end{array}$ & $\beta$ & $\begin{array}{l}B(S E) \\
n=75\end{array}$ & $\beta$ & $\begin{array}{l}B(S E) \\
n=75\end{array}$ & $\beta$ & $\begin{array}{l}B(S E) \\
n=75\end{array}$ & $\beta$ \\
\hline $\begin{array}{l}\text { Sex } \\
n=75\end{array}$ & $-.55(.50)$ & -.16 & $1.03(4.22)$ & .03 & $-.19(1.15)$ & -.02 & $.12(1.01)$ & .02 & $.02(.32)$ & .01 \\
\hline $\begin{array}{l}\text { Age at testing } \\
n=75\end{array}$ & $-.02(.08)$ & -.03 & $-.26(.68)$ & -.05 & $.20(.18)$ & .13 & $.07(.16)$ & .05 & $-.009(.05)$ & -.02 \\
\hline $\begin{array}{l}\text { Time since last injury } \\
n=61\end{array}$ & $-.001(.01)$ & -.02 & $-.11(.11)$ & -.14 & $-.02(.03)$ & -.10 & $.04(.03)$ & .21 & $.005(.009)$ & .09 \\
\hline $\begin{array}{l}\# \text { of concussions }{ }^{\mathrm{b}} \\
n=75\end{array}$ & $-1.54(.98)$ & -.23 & $-7.15(8.25)$ & -.11 & $3.91(2.25)$ & .24 & $.68(1.97)$ & .05 & $-.06(.63)$ & -.01 \\
\hline $\begin{array}{l}\text { Pre-attention } \\
n=75\end{array}$ & $.02(.62)$ & .004 & $-11.32(5.27)$ & -.30 & $2.52(1.43)$ & .25 & $1.88(1.26)$ & .21 & $.47(.40)$ & .18 \\
\hline $\begin{array}{l}\text { Pre-learning } \\
n=75\end{array}$ & $.55(.68)$ & .12 & $-5.38(7.52)$ & .22 & $-1.03(1.57)$ & -.09 & $-2.57(1.37)$ & -.26 & $-.41(.44)$ & -.14 \\
\hline $\begin{array}{l}\text { Pre-mood } \\
n=74\end{array}$ & $-1.36(.79)$ & -.29 & $-14.90(6.66)$ & -.35 & $3.45(1.81)$ & .31 & $2.65(1.59)$ & .26 & $1.15(.51)$ & .38 \\
\hline $\begin{array}{l}\text { Pre-anxiety } \\
n=74\end{array}$ & $.03(.63)$ & .007 & $-2.67(5.28)$ & -.08 & $.28(1.44)$ & .03 & $1.63(1.26)$ & .20 & $-.19(.41)$ & -.08 \\
\hline $\begin{array}{l}\text { Pre-migraines } \\
n=75\end{array}$ & $-.78(1.11)$ & -.10 & $-1.67(9.33)$ & -.02 & $2.77(2.54)$ & .15 & $-.96(2.22)$ & -.06 & $-.26(.72)$ & -.05 \\
\hline$R^{2}$ & .174 & & .305 & & .271 & & .290 & & .172 & \\
\hline$F$ & 1.174 & & 2.439 & & 2.060 & & 2.266 & & 1.152 & \\
\hline
\end{tabular}

Notes. PedsQL $=$ Pediatric Quality of Life Inventory; $B=$ unstandardized regression coefficients; $S E=$ standard error; $\beta=$ standardized regression coefficients; $R^{2}=$ multiple correlation squared; $F=F$ distribution; $n=$ number of cases.

${ }^{a}$ Square root transformed scores used.

${ }^{\mathrm{b}}$ Logarithmic transformed scores used; $* p<.01$. 
or health-related quality of life. These results contrast with previous studies showing recurrent concussions as a predictor of psychological problems (Stazyk et al., 2017) and health-related quality of life (Novak et al., 2016). However, these previous studies were conducted on average within 3 months after injury, which might explain why previous results differ from the present study. Future studies with bigger samples of children and adolescents who experienced multiple concussions are needed to replicate the current findings and to determine if the number of concussions is a significant predictor of psychosocial functioning and healthrelated quality of life only early after injury.

Age and sex of the participants, the time since the last injury, and most pre-existing conditions were also not significant predictors of psychosocial functioning or health-related quality of life. The only exception was that pre-existing attention problems were a significant predictor of psychosocial functioning (more specifically, attention/hyperactivity and conduct problems) while pre-existing mood issues were a significant predictor of depressive symptoms. These pre-existing conditions appear to play a more important role in predicting long-term functioning than concussion itself (Brooks et al., 2018).

Alternatively, some researchers have suggested that pre-existing conditions could impact the ability to cope after brain injuries (Barlow, 2016; Bernard et al., 2016) or could be viewed as predisposing factors of a slower recovery post-concussion. Despite these significant results, our study did not identify factors significantly predicting long-term health-related quality of life. Other factors not included in our analyses, such as current mental health status, could play a more important role in determining health-related quality of life than pre-existing conditions.

The current study has some limitations that temper its conclusions. First, the small number of participants may have reduced statistical power and hindered the detection of differences between groups or of significant outcome predictors, including pre-existing conditions reported by parents. For instance, limited power might partly explain why preexisting mood concerns did not significantly predict healthrelated quality of life despite a medium effect size. Moreover, families who agreed to return to the hospital for a research project after such a lengthy time post-injury may have differed from those who would not do so; this may represent a selection bias and limit generalizability.

In addition, the study design was cross-sectional, which could lead to retrospective bias when parents were asked report on their child's pre-injury status. In a previous study on the "good old days bias" (Brooks et al., 2014), parents' ratings appeared to underestimate their children's pre-injury difficulties. Future studies using a prospective longitudinal design and a bigger sample size are needed to address these limitations.

Despite these limitations, the study helps to address growing concerns regarding the potential long-term consequences associated with multiple concussions in youth and to better understanding which factors should be addressed when working clinically with children and adolescents who report lingering difficulties. Overall, the results underscore the clinical importance of asking about pre-injury problems, especially those related to attention and mood, as they may predict longer term psychosocial functioning in children and adolescents better than concussion itself. The findings also reinforce the importance of future studies on long-term outcomes in youth to better understand the ramification of recurrent concussions.

\section{ACKNOWLEDGMENTS}

Special thanks to the families who participated in this research. The authors thank the members of the NEURO-detect study team (Karen M. Barlow, MB.ChB. MRCPCH (UK), MSc, Helen Carlson, $\mathrm{PhD}$, Michael Esser, MD, MSc, Kathryn Schneider, PhD, PT, Catherine Lebel, PhD, Signe Bray, PhD, Ashley Harris, PhD, Marc Lebel, PhD, and Frank P. MacMaster, PhD). Thanks to Zeanna Jadavji, BSc (Hons), Trevor A. Low, BSc (Hons), and Shane Virani, MSc for assistance with recruitment, data collection, and data entry. Thanks to Lonna Mitchell, BA and Kalina Slepicka, BA for scoring protocols and (alphabetically) Amy Bobyn, Dominique Bonneville, BA (Hons), Shauna Bulman, Christianne Laliberté-Durish, MSc, Shelby MacPhail, Maya Sohn, and Cole Sugden for assistance with data entry. Thanks to Brenda Turley, BA (Hons), Carolyn Emery, $\mathrm{PhD}$ PT, and Kathryn Schneider, PhD PT for assisting with recruitment. Recruitment of some control participants was done using the Healthy Infants and Children Clinical Research Program database (HICCUP; http://www.ucalgary.ca/paediatrics_hiccup). Disclosure Brian Brooks receives royalties from Psychological Assessment Resources Inc. for pediatric neuropsychological tests [Child and Adolescent Memory Profile (ChAMP), Sherman \& Brooks, 2015; Memory Validity Profile (MVP), Sherman \& Brooks, 2015; Multidimensional Everyday Memory Ratings for Youth (MEMRY), Sherman \& Brooks, 2017], and from Oxford University Press [Sherman \& Brooks, 2012; Pediatric Forensic Neuropsychology]. He has previously received in-kind support (free test credits for prior studies) from the publisher of a computerized cognitive test (CNS Vital Signs, Chapel Hill, North Carolina). Keith Yeates receives royalties for book sales from Guilford Press and Cambridge University Press, and occasionally serves as a paid expert in forensic cases. Vickie Plourde does not have any conflicts of interest to declare. None of the authors have a financial interest in any measures used in the present study. Funding This study was funded by the Shaikh Family Research Award, an endowment held by the Alberta Children's Hospital Foundation and granted to Brian Brooks by the Alberta Children's Hospital Research Institute. Additional support for the study was provided from the Ronald and Irene Ward Chair in Pediatric Brain Injury (awarded to Keith Yeates). Brian Brooks acknowledges salary funding from the Canadian Institutes for Health Research (CIHR) Embedded Clinician Researcher Salary Award. Vickie Plourde acknowledges postdoctoral fellowship funding from the Alberta Children's Hospital Research Institute (University of Calgary), the Integrated Concussion Research Program (University of Calgary), and Alberta Innovates-Health Solutions.

\section{REFERENCES}

Barker-Collo, S.L. (2007). Behavioural profiles and injury severity following childhood traumatic brain injury. Brain Impairment, 8, 22-30. http://doi.org/10.1375/brim.8.1.22 
Barlow, K.M. (2016). Postconcussion dyndrome: A review. Journal of Child Neurology, 31, 57-67. http://doi.org/10.1177/ 0883073814543305

Barlow, K.M., Crawford, S., Stevenson, A., Sandhu, S.S., Belanger, F., \& Dewey, D. (2010). Epidemiology of postconcussion syndrome in pediatric mild traumatic brain injury. Pediatrics, 126(2), e374-e381. http://doi.org/10.1542/peds.2009-0925

Basson, M.D., Guinn, J.E., McElligott, J., Vitale, R., Brown, W., \& Fielding, P. (1991). Behavioral disturbances in children after trauma. The Journal of Trauma, 31(10), 1363-1368.

Bernard, C.O., Ponsford, J.A., McKinlay, A., McKenzie, D., \& Krieser, D. (2016). Predictors of post-concussive symptoms in young children: Injury versus non-injury related factors. Journal of the International Neuropsychological Society, 22, 793-803. http://doi.org/10.1017/S1355617716000709

Bøe, T., Hysing, M., Skogen, J.C., \& Breivik, K. (2016). The Strengths and Difficulties Questionnaire (SDQ): Factor structure and gender equivalence in Norwegian adolescents. PLoS One, 11(5), 1-15. http://doi.org/10.1371/journal.pone.0152202

Bourdon, K.H., Goodman, R., \& Rae, D.S. (2005). The Strengths and Difficulties Questionnaire: U.S. normative data and psychometric properties. Journal of the American Academy of Child \& Adolescent Psychiatry, 44(6), 557-564. http://doi.org/10.1097/ 01.chi.0000159157.57075.c8

Brooks, B.L., Kadoura, B., Turley, B., Crawford, S., Mikrogianakis, A., \& Barlow, K.M. (2014). Perception of recovery after pediatric mild traumatic brain injury is influenced by the "good old days" bias: Tangible implications for clinical practice and outcomes research. Archives of Clinical Neuropsychology, 29, 186-193. http://doi.org/10.1093/arclin/act083

Brooks, B.L., Silverberg, N., Maxwell, B., Mannix, R., Zafonte, R., Berkner, P.D., \& Iverson, G.L. (2018). Investigating effects of sex differences and prior concussions on symptom reporting and cognition among adolescent soccer players. The American Journal of Sports Medicine, advanced online publication. http:// doi.org/10.1177/0363546517749588

Catroppa, C., Hearps, S., Crossley, L., Yeates, K., Beauchamp, M., Fusella, J., \& Anderson, V. (2017). Social and behavioral outcomes following childhood traumatic brain injury: What predicts outcomes at 12 months post-insult. Journal of Neurotrauma, 34, 1439-1447. http://doi.org/10.1089/neu. 2016.4594

Cohen, J. (1992). A power primer. Psychological Bulletin, 112(1), 155-159. http://doi.org/10.1037/0033-2909.112.1.155

Curvis, W., Mcnulty, S., \& Qualter, P. (2014). The validation of the self-report strengths and difficulties questionnaire for use by 6- to 10-year-old children in the UK. British Journal of Clinical Psychology, 53, 131-137. http://doi.org/10.1111/bjc.12025

Davis, G.A., Anderson, V., Babl, F.E., Gioia, G.A., Giza, C.C., Meehan, W., ... Zemek, R. (2017). What is the difference in concussion management in children as compared with adults? A systematic review. British Journal of Sports Medicine, 1-12. http://doi.org/10.1136/bjsports-2016-097415

Emery, C.A., Barlow, K.M., Brooks, B.L., Max, J.E., VillavicencioRequis, A., Gnanakumar, V., ... Yeates, K.O. (2016). A systematic review of psychiatric, psychological, and behavioural outcomes following mild traumatic brain injury in children and adolescents. Canadian Journal of Psychiatry, 61(5), 259-269. http://doi.org/10.1177/0706743716643741

Goodman, R. (1997). The Strengths and Difficulties Questionnaire: A research note. Journal of Child Psychology and Psychiatry, 38(5), 581-586.
Goodman, R. (2001). Psychometric properties of the Strengths and Difficulties Questionnaire. Journal of the American Academy of Child \& Adolescent Psychiatry, 40(11), 1337-1345. http://doi. org/10.1097/00004583-200111000-00015

Goodman, R., Meltzer, H., \& Bailey, V. (1998). The strengths and difficulties questionnaire: A pilot study on the validity of the self-report version. European Child and Adolescent Psychiatry, 130, 125-131.

Grubenhoff, J.A., Currie, D., Dawn Comstock, R., Juarez-Colunga, E., Bajaj, L., \& Kirkwood, M.W. (2016). Psychological factors associated with delayed symptom resolution in children with concussion. Journal of Pediatrics, 174, 27-32.e1. http://doi.org/ 10.1016/j.jpeds.2016.03.027

Hawley, C.A., Ward, A.B., Magnay, A.R., \& Long, J. (2004). Outcomes following childhood head injury: A population study. Journal of Neurology, Neurosurgery, and Psychiatry, 75(5), 737-742. http://doi.org/10.1136/jnnp.2003.020651

IBM Corporation. (2013). IBM SPSS Statistics for Macintosh. Armonk, NY: IBM Corporation.

Institute of Medicine \& National Research Council. (2014). Sportsrelated concussions in youth: Improving the science, changing the culture. In R. Graham, F.P. Rivara, M.A. Ford, \& C.M. Spicer, Eds. Washington, DC: The National Academies Press.

Iverson, G.L. (2010). Mild traumatic brain injury meta-analyses can obscure individual differences. Brain Injury, 24(10), 1246-1255. http://doi.org/10.3109/02699052.2010.490513

Iverson, G.L., Gardner, A.J., Terry, D.P., Ponsford, J.L., Sills, A.K., Broshek, D.K., \& Solomon, G.S. (2017). Predictors of clinical recovery from concussion: A systematic review. British Journal of Sports Medicine , 51, 941-948. http://doi.org/10.1136/bjsports2017-097729

Iverson, G.L., \& Schatz, P. (2015). Advanced topics in neuropsychological assessment following sport-related concussion. Brain Injury, 29(2), 263-275. http://doi.org/10.3109/02699052.2014.965214

Lovell, M.R., Iverson, G.L., Collins, M.W., Podell, K., Johnston, K.M., Pardini, D., ... Maroon, J.C. (2006). Measurement of symptoms following sports-related concussion: Reliability and normative data for the Post-Concussion Scale. Applied Neuropsychology, 13(3), 3166-3174. http://doi.org/10.1207/s15324826an1303

Luis, C.A., \& Mittenberg, W. (2002). Mood and anxiety disorders following pediatric traumatic brain injury: A prospective study. Journal of Clinical \& Experimental Neuropsychology, 24(3), 270-279.

Max, J.E., Lansing, A.E., Koele, S.L., Castillo, C.S., Bokura, H., Schachar, R., ... Williams, K.E. (2004). Attention deficit hyperactivity disorder in children and adolescents following traumatic brain injury. Developmental Neuropsychology, 25(1-2), 159-177. http://doi.org/10.1080/87565641.2004.9651926

Mccauley, S.R., Wilde, E.A., Anderson, V.A., Bedell, G., Beers, S.R., Campbell, T.F., ... Yeates, K.O. (2012). Recommendations for the use of common outcome measures in pediatric traumatic brain injury research. Journal of Neurotrauma, 29(4), 678-705. http://doi.org/ 10.1089/neu.2011.1838

McNally, K.A., Bangert, B., Dietrich, A., Nuss, K., Rusin, J., Wright, M., ... Yeates, K.O. (2013). Injury versus non-injury factors as predictors of post-concussive symptoms following mild traumatic brain injury in children. Neuropsychology, 27(1), 1-12. http://doi.org/10.1037/a0031370.Injury

Merritt, V.C., \& Arnett, P.A. (2014). Premorbid predictors of postconcussion symptoms in collegiate athletes. Journal of Clinical and Experimental Neuropsychology, 36(10), 1098-1111. http://doi. org/10.1080/13803395.2014.983463 
Moran, L.M., Taylor, H.G., Rusin, J., Bangert, B., Dietrich, A., Nuss, K.E., ... Yeates, K.O. (2012). Quality of life in pediatric mild traumatic brain injury and its relationship to postconcussive symptoms. Journal of Pediatric Psychology, 37(7), 736-744.

Morgan, C.D., Zuckerman, S.L., Lee, Y.M., King, L., Beaird, S., Sills, A.K., \& Solomon, G.S. (2015). Predictors of postconcussion syndrome after sports-related concussion in young athletes: A matched case-control study. Journal of Neurosurgery. Pediatrics, 15(6), 589-598. http://doi.org/10.3171/2014.10. PEDS14356.Disclosure

Muris, P., Meesters, C., Eijkelenboom, A., \& Vincken, M. (2004). The self-report version of the Strengths and Difficulties Questionnaire: Its psychometric properties in 8- to 13-year-old non-clinical children. British Journal of Clinical Psychology, 43, 437-448.

Novak, Z., Aglipay, M., Barrowman, N., Yeates, K.O., Beauchamp, M.H., Gravel, J., ... Zemek, R.L. (2016). Association of persistent postconcussion symptoms with pediatric quality of life. JAMA Pediatrics, E1-E8. http://doi.org/10.1001/ jamapediatrics.2016.2900

O'Connor, S.S., Zatzick, D.F., Wang, J., Temkin, N., Koepsell, T.D., Jaffe, K.M., ... Rivara, F.P. (2012). Association between posttraumatic stress, depression, and functional impairments in adolescents 24 months after traumatic brain. Journal of Traumatic Stress, 25, 264-271. http://doi.org/10.1002/jts.21704

Ponsford, J., Willmott, C., Rothwell, A., Cameron, P., Ayton, G., Nelms, R., ... Ng, K.T. (1999). Cognitive and behavioral outcome following mild traumatic head injury in children. The Journal of Head Trauma Rehabilitation, 14(4), 360-372.

Reynolds, C.R., \& Kamphaus, R.W. (2004). BASC-2: Behavior assessment system for children. Circle Pines, MN: American Guidance Service.

Root, J.M., Zuckerbraun, N.S., Wang, L., Winger, D.G., Brent, D., Kontos, A., \& Hickey, R.W. (2016). History of somatization is associated with prolonged recovery from concussion. Journal of Pediatrics, 174, 39-44.e1. http://doi.org/10.1016/j.jpeds.2016.03.020

Roy, B. Van, Veenstra, M., \& Clench-Aas, J. (2008). Construct validity of the five-factor Strengths and Difficulties Questionnaire (SDQ) in pre-, early, and late adolescence. Journal of Child Psychology and Psychiatry, 49(12), 1304-1312. http://doi.org/ 10.1111/j.1469-7610.2008.01942.x

Russell, K., Selci, E., Chu, S., Fineblit, S., Ritchie, L., \& Ellis, M.J. (2017). Longitudinal assessment of health-related quality of life following adolescent sports-related concussion. Journal of Neurotrauma, 34, 2147-2153. http://doi.org/10.1089/neu.2016.4704

Sady, M.D., Vaughan, C.G., \& Gioia, G.A. (2014). Psychometric characteristics of the postconcussion symptom inventory in children and adolescents. Archives of Clinical Neuropsychology, 29(4), 348-363. http://doi.org/10.1093/arclin/acu014

Sariaslan, A., Sharp, D.J., Onofrio, B.M.D., Larsson, H., \& Fazel, S. (2016). Long-term outcomes associated with traumatic brain injury in childhood and adolescence: A nationwide Swedish
Cohort Study of a wide range of medical and social outcomes. PLoS Medicine, 15-19. http://doi.org/10.1371/journal.pmed. 1002103

Solomon, G.S., Kuhn, A.W., \& Zuckerman, S.L. (2016). Depression as a modifying factor in sport-related concussion: A critical review of the literature. The Physician and Sportsmedicine, 44(1), 14-19. http://doi.org/10.1080/00913847.2016.1121091

Stazyk, K., DeMatteo, C., Moll, S., \& Missiuna, C. (2017). Depression in youth recovering from concussion: Correlates and predictors. Brain Injury, 1-8. http://doi.org/10.1080/02699052.2017.1283533

Varni, J.W., Seid, M., \& Kurtin, P.S. (2001). PedsQL ${ }^{\mathrm{TM}} 4.0$ : Reliability and validity of the Pediatric Quality of Life Inventory ${ }^{\mathrm{TM}}$ Version 4.0 Generic Core Scales in Healthy and Patient. Medical Care, 39(8), 800-812.

Varni, J.W., Burwinkle, T.M., Seid, M., \& Skarr, D. (2003). The PedsQLTM 4.0 as a pediatric population health measure: Feasibility, reliability, and validity. Ambulatory Pediatrics, 3(6), 329-341.

Varni, J.W., Seid, M., \& Rode, C.A. (1999). The PedsQL ${ }^{\mathrm{TM}}$. Measurement model for the pediatric quality of life inventory. Medical Care, 37(2), 126-139.

Yeates, K.O., Kaizar, E., Rusin, J., Bangert, B., Dietrich, A., Nuss, K., ... Taylor, H.G. (2012). Reliable change in postconcussive symptoms and its functional consequences among children with mild traumatic brain injury. Archives of Pediatrics \& Adolescent Medicine, 166(7), 615-622. http://doi.org/10.1001/ archpediatrics.2011.1082

Yeates, K.O., Luria, J., Bartkowski, H., Rusin, J., Martin, L., \& Bigler, E.D. (1999). Postconcussive symptoms in children with mild closed head injuries. Journal of Head Trauma Rehabilitation, 14(4), 337-350.

Yeates, K.O., Taylor, H.G., Rusin, J., Bangert, B., Dietrich, A., Nuss, K., \& Wright, M. (2012). Premorbid child and family functioning as predictors of post-concussive symptoms in children with mild traumatic brain injuries. International Journal of Developmental Neuroscience, 30(3), 231-237. http://doi.org/ 10.1016/j.ijdevneu.2011.05.008

Zemek, R., Barrowman, N., Freedman, S.B., Gravel, J., Gagnon, I., McGahern, C., ... Pediatric Emergency Research Canada (PERC) Concussion Team. (2016). Clinical risk score for persistent postconcussion symptoms among children with acute concussion in the ED. Journal of the American Medical Association, 315(10), 1014-1025. http://doi.org/10.1001/ jama.2016.1203

Zemek, R.L., Farion, K.J., Sampson, M., \& McGahern, C. (2013). Prognosticators of persistent symptoms following pediatric concussion: A systematic review. JAMA Pediatrics, 167(3), 259-265. http://doi.org/10.1001/2013.jamapediatrics.216

Zuckerman, S.L., Yengo-Kahn, A.M., Buckley, T.A., Solomon, G.S., Sills, A.K., \& Kerr, Z.Y. (2016). Predictors of postconcussion syndrome in collegiate student-athletes. Neurosurgical Focus, 40(4). http://doi.org/10.3171/2016.1.FOCUS15593 